\title{
SIMULTANEOUS DOA ESTIMATION BASED ON KOLMOGOROV'S THEOREM*
}

\author{
Montse Nájar, Miguel A. Lagunas
}

\author{
ETSI. de Telecomunicación, TSC Department \\ Apdo. 30002, 08080 BARCELONA, SPAIN
}

\begin{abstract}
The design of a new architecture for signal processing, based on the Kolmogorov's Theorem, is addressed in this paper. This architecture is applied to solve the problem of source separation. Particularly, an adaptive algorithm is proposed to separate simultaneously all the unknown impinging sources on an aperture of sensors. The implemented framework is composed of two different stages: the first one is the inhibition stage, wich turns the problem of estimating simultaneous DOAs (directions of arrival) into problems of a single source DOA estimation; and the second one is the optimisation stage which estimates the required parameter in a single signal context easier than the initial one with multiple signal. A high order rule for learning is described, it improves the behaviour of the system assuring independence of the outputs.
\end{abstract}

\section{INTRODUCTION}

In 1957 Kolmogorov proved that any continuous multiple variable function can be represented by superpositions of functions of a single variable. From that time, several interpretations of this theorem have been done, specially from the Neural Network viewpoint [2], [3], [8], [9] and [10]. Unfortunately, no especific examples of implementation of this theorem are known.

The theorem has been evolved to a processing framework including the Neural Network Architectures, the Estimate-Maximise (EM) Algorithm and the potential of the High Order for learning. Also it is important to highlight the independence of the resulting network from the specific mapping required.

This paper deals with the so-called 'source separation' problem. Because of its numerous possible applications, in particular in the framework of antenna array processing, the attention paid to this subject has recently increased.

Following the pioneer task in the source separation problem by Common, Herault and Jutten [1], [4] and [5], among others, some schemes have been developed for multitone tracking and DOA estimation, based on the Kolmogorv's Theorem.

This paper will focus on the DOA estimation problem. Quite often the situation may be described as several sources in different directions, radiating at the same time, which impinge on an aperture of sensors. The signal received by each sensor is usually disturbed by noise from the amplifiers of the sensors. Then, the input of the processor will be a set of narrowband array snapshots. It is possible to find out the direction of arrival of the impinging sources, by means of any of the well-known array processing techniques; for instance: Music, Esprit, etc. Applying the Kolmogorov's Theorem it is possible to decompose the problem of multisource scenario in several problems of only one source.

First of all we will outline the Kolmogorov's Theorem: next, an approach relying on this theorem is proposed to solve the problem of simultaneous DOA estimation and source beamforming in narrowband array processing, where a new technique of high order learning is included: finally, some simulation results will be shown.

\section{KOLMOGOROV'S THEOREM}

In 1957 Kolmogorov published [7] the Mapping Neural Network Existence Theorem. This theorem concems the representation of arbitrary continuous functions of $n$ variables by sums and superpositions of continuous functions of only one variable. Important references of this theorem are the works by Lorentz [14] and by HechtNielsen [3]. The last summarised this theorem as:

Given any continuous function $f(x)=y$, where $x$ and $y$ are vectors with $n$ and $m$ components respectively, this function can be implemented by a two-stage network. The first stage produces $2 n+1$ outputs and the second stage provides the desired $m$ components of vector $y$.

The total transfer function can be formulated as:

$$
y_{i}=\sum_{k=1}^{2 n+1} g_{i}\left(\sum_{j=1}^{n} \varphi_{k}\left(x_{j}\right)\right)
$$

where the design of the $2 n+1$ functions $\varphi_{k}$, forming the first stage, is independent of $f$, although it depends on $n$. And the $m$ functions $g_{i}$, forming the second stage, are real and continuous. figure 1.

An illustration of this theorem can be viewed in

It is important to notice that all these functions depend on a single variable. Thus, this processor turns the multivariate problem into several single variable ones.

Some examples of applications of this processor have been reported in [15]. In this paper, we are going to

*This work was supported by the National Research Plan of Spain, CICYT, Grant number TIC92-0800-C05-05. 
focus only on the Kolmogorov's Signal Processor for Separation of Sources.

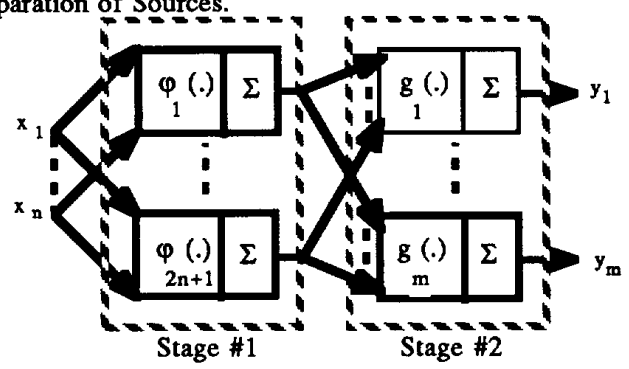

Figure 1. The Kolmogorov's Signal Processor

\section{SIGNAL PROCESSOR FOR SEPARATION OF SOURCES}

A processor based on the above described theorem has been developed [13], [15] and [16] in order to solve the problem of simultaneous DOA estimation in Array Processing. Let's imagine a multiple source scenario where the direction of arrival of each source is unknown. At the input of each sensor a mixture of the different signals can be measured, then, a multiparameter estimation is needed. We decompose this problem into easier problems of a single parameter estimation, by means of this new processor.

The aforementioned Kolmogorov's processor is constituted of two different stages. From now on we will call them the inhibition and the optimisation stages. In order to gain an insight into the system, we will consider it divided into branches, one branch for each impinging source.

With the purpose of describing clearly the resultant framework, we will focus on the problem of only two sources present in the array scenario; nevertheless, the obtained architecture can be easily generalised to the case of any number of sources. Then, assuming this case, a two branch system is needed, each of them has to estimate the wanted parameter of one of the sources. The inhibition stage will turn the problem into two problems of single source DOA estimation. The optimisation stage of each branch will estimate one of the two DOAs $\left(\theta_{1}, \theta_{2}\right)$. An adaptive learning of the first stage from the second one, assures the convergence of the algorithm. in figure 2 :

This system can be represented by the architecture

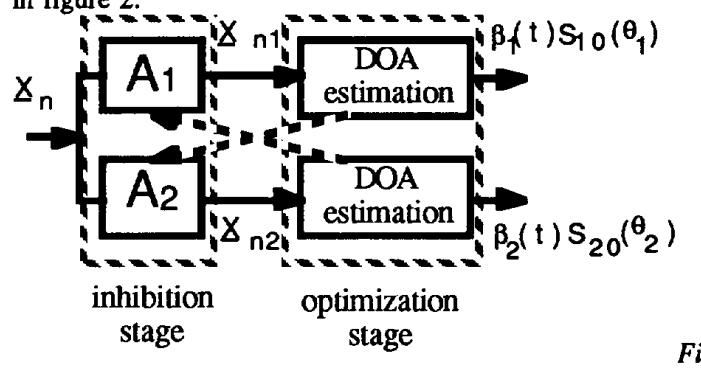

gure 2. Two stage network for source DOA estimation

\subsection{The Inhibition Stage}

The first stage of our approach to the Kolmogorov's Theorem has been named the inhibition stage. There exist two alternatives for the inhibition scheme: the direct and the indirect inhibition.

Direct inhibition consists in the direct subtraction of the second stage output of one branch at the input of the other. A previous work with this kind of inhibition was the scheme reported by Herault, Jutten and Common [1], [4]. Two examples of direct inhibition we have worked on are: the first, a multitone tracker using Extended Kalman Filters (EKF) [11], and the second, a system to estimate the source DOAs in Array Processing, also with EKFs [12] and [16].

This paper will concentrate on the indirect inhibition, developed in [15]. This inhibition stage uses a blocking matrix in each branch which eliminates the influence of one of the input sources.

For the narrowband case the blocking matrix at the first branch can be formed as follows, being $Q$ the number of array sensors:

$$
\begin{gathered}
A_{1}=\left(\begin{array}{cccccc}
1 & -\alpha_{1} & 0 & \ldots & 0 \\
0 & 1 & -\alpha_{2} & 0 & \checkmark \\
\vdots & 0 & \checkmark & \checkmark & 0 \\
0 & 0 & 0 & \ldots & 1 & -\alpha_{q-1}
\end{array}\right) \\
\alpha_{q}=\exp \left(\frac{j 2 \pi f_{c}}{c}\left(d_{q} \cos \varphi_{q}-d_{q+1} \cos \varphi_{q+1}\right) \sin \theta_{2}\right.
\end{gathered}
$$

The angle of arrival of the source to be blocked by the inhibition matrix, $\Theta_{2}$, is obtained by the output of the second branch.

Let us suppose a linear equally spaced array, then all the $\alpha_{i}$ are equal and have the simple expression:

$$
\alpha=\exp \left(-\mathrm{j} \frac{2 \pi \mathrm{f}_{\mathrm{c}}}{\mathrm{c}} \mathrm{d} \sin \theta_{2}\right)
$$

Two drawbacks of this scheme, that easily can be overcome, are:

First, the dimension of the original steering vector is reduced from the number of sensors to the number of sensors minus one.

$$
\underline{S}_{1}^{\prime}=\underline{A} \underline{S}_{1}
$$

A simple recurrent equation allows us to recover the original steering from the modified one:

$$
\begin{gathered}
S_{1}(0)=1 \\
S_{1}(n)=\frac{S_{1}(n-1)-S_{1}^{\prime}(n)}{\alpha_{n}}
\end{gathered}
$$

Secondly, this matrix produces a coloured noise background that makes difficulties to the estimation at the second stage. We will see in the next section how to solve this problem.

In spite of these disadvantages we have proved the indirect inhibition to be more useful than the direct one.

\subsection{The Optimisation Stage}

The second stage of our processor has been called the optimisation stage. This stage is devoted to the estimation of the source parameters. If the inhibition has 
been done in a proper way, the input of the optimisation stage of each branch will have only information about one source. Then, the process of estimation will be easy, but it will depend on the applied inhibition stage.

In the case of direct inhibition, a estimation of the waveforms is needed, it can be done with Extended Kalman Filters. Some results of this system can be seen in [16].

In the case this paper is dealing with, indirect inhibition, the single parameter required is the DOA of each source. Thus, a simple spectral analysis is adequate for the optimisation stage. For instance, it is well-known that the signal subspace can be estimated by the NS (number of sources) eigenvalues of the array correlation matrix. Thus, if a single source is present at the input of this stage, the eigenvector corresponding to the greatest eigenvalue must be equal to the steering vector of this source. And it will be possible to find the DOA from the maximum of the resulting beamforming:

$$
\left|S_{i}{ }_{i}^{H}{ }_{\max }\right|^{2}
$$

where each $S_{\mathfrak{i}}$ is the steering vector of a possible direction of arrival and $e_{\max }$ the greatest eigenvector.

Because the system works in the aforementioned coloured noise background, the correlation matrix is given by:

$$
\underline{R}_{x x}=\underline{A} S \underline{R}_{S S} S^{H} \underline{A}^{H}+\sigma^{2} \underline{\underline{A}} \underline{A}^{H}
$$

where $A$ denotes the blocking matrix and $R_{S S}$ is the correlation matrix of the input signals.

In this case the vector we need is:

$$
\underline{\underline{A}} \stackrel{\text { A }}{{ }^{\mathbf{e}}} \text {, }
$$

being $e_{1}$ the generalised eigenvector (GEV) of $R_{\mathbf{X x}}$ corresponding to the largest generalised eigenvalue (GE). reported in [15]

Some results yielded by this system have been

\section{HIGH ORDER LEARNING}

The scheme developed in [11] formed by direct inhibition and EKF presents a problem, due to the system symmetry, the two resulting waveforms could be equal. With the purpose of avoiding that, this system has to make the outputs, $y_{1}(n)$ and $y_{2}(n)$, as orthogonal as possible, controlling the evolution of the two adaptive weights, $a_{12}(n)$ and $a_{21}(n)$.

This scheme is depicted in the next figure:

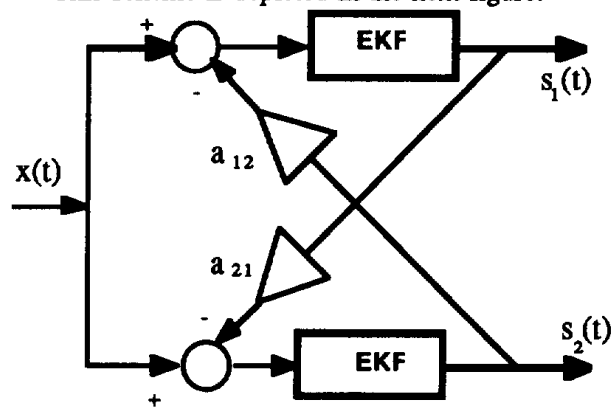

Figure 3 Scheme with direct inhibition
In order to achieve this orthogonality, it is necessary to minimise the scalar product between both outputs in a given time interval $\mathrm{T}$

$$
\xi=\left[\int_{r} y_{1}(t) y_{2}^{*}(t) d t\right]^{2}
$$

The learning rule is obtained from the derivative of this expression with respect to each weight. Taking the instantaneous value of the integral, assuming a small interval of time $T$, and using the gradient method, the weights are updated as:

$$
\begin{aligned}
& a_{12}(n+1)=a_{12}(n)+\mu_{1}\left|y_{2}\right|^{2} y_{1} y_{2}^{*} \\
& a_{21}(n+1)=a_{21}(n)+\mu_{2}\left|y_{1}\right|^{2} y_{2} y_{1}^{*}
\end{aligned}
$$

It is important to notice the high order character of the correction term. This kind of leaming has been used previously in [5] and [6].

In the scheme we are carrying out in this paper, with indirect inhibition and spectral estimation, the outputs are the source DOAs.

The criterion for the design of the learning rule in this case, can be to achieve independent perturbations: $\tilde{\theta}_{1}$ and $\tilde{\theta}_{2}$, defined as the differences between the current angle values at the inhibition matrix and the corresponding average mean of the previous values:

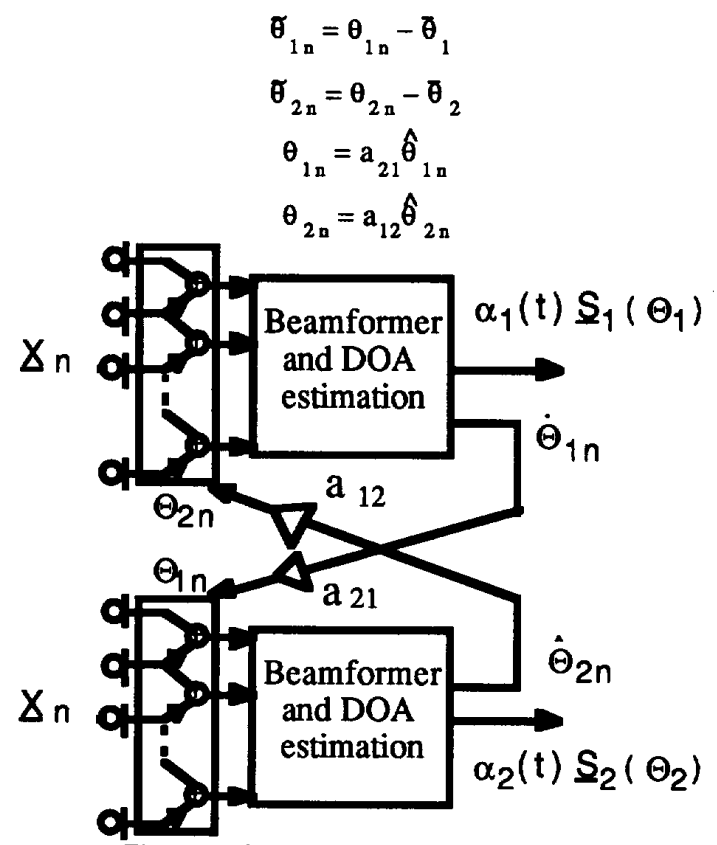

Figure 4. Scheme with indirect inhibition

The perturbation independence can be obtained minimising this expression:

$$
\zeta=E\left[\begin{array}{lll}
\theta_{1 \mathrm{n}} & \theta_{2 \mathrm{n}}
\end{array}\right]^{2}
$$

Taking again the instantaneous value and deriving with respect to each weight: 


$$
\begin{aligned}
& \frac{d \zeta}{d a_{12}}=\theta_{1 n}^{2} \theta_{2 n} \frac{d \theta_{2 n}}{d a_{12}}=\theta_{1 n}^{2} \theta_{2 n} \hat{\theta}_{2 n} \\
& \frac{d \zeta}{d a_{21}}=\theta_{2 n}^{2} \theta_{1 n} \frac{d \theta_{1 n}}{d a_{21}}=\theta_{2 n}^{2} \theta_{1 n} \hat{\theta}_{1 n}
\end{aligned}
$$

The learning rule is obtained:

$$
\begin{aligned}
& a_{12}(n+1)=a_{12}(n)+\mu_{1} \theta_{1 n}^{2} \theta_{2 n} \hat{\theta}_{2 n} \\
& a_{21}(n+1)=a_{21}(n)+\mu_{2} \theta_{2 n}{ }^{\theta_{1 n}} \hat{\theta}_{1 n}
\end{aligned}
$$

In the next section, some results will show the improvements of this high order leaming.

\section{SIMULATION RESULTS}

The presented simulations have been made with a linear equally spaced array of 8 sensors, in which the interelement separation was half wavelength.

In the simulations where the scenario is formed by two sources (with a SNR of $3 \mathrm{~dB}$ each of them), problems did not appear whether the high order learning was applied or not. Each branch estimates one of the two different sources. In figures 5.a and 5.b, the evolution of the estimated angles and the beamformer at the last iteration can be seen. In this simulation, the scenario was formed by two moving sources which cross each other.

Problems appear when only one source is present in the scenario. Without high order learning the two system branches estimate close angles which cross each other sometimes. Because of the proximity of the two estimated angles, the estimated power at the two branches are very low. This effect can be observed in figures 6.a and 6.b.

However, when high order learning is applied, one of the two branches estimates the correct angle, with high power level, and the other escapes to a far-off angle. They never cross each other. Figures 7.a and 7.b show the results of this simulation.

\section{CONCLUSIONS}

A new signal processor based on the so-called Kolmogorov's Theorem has been reported.

This processor has been successfully applied to the problem of DOA estimation in a multisource scenario. Although the analysis has been restricted to the case of only two sources, in order to reduce complexity, the system can be easily extended to the case of any number of sources.

A high order leaming has been included in the scheme, this new technique improves the behaviour of the system.

Other possible applications of this signal processor are being investigated by the authors.

\section{References}

[1] Common P. Jutten C. , Herault J., "Blind separation of sources Part II: Problem statement", Signal Processing, vol 24, $\mathrm{n}^{2} 1$, pp. 11-21, July 1991.

[2] Girosi F. , Poggio T. , "Representation Properties of Networks: Kolmogorov's Theorem is Irrevelant",
Neural Computation (Winter 1989), vol. 1, no.4, pp 465-469.

[3] Hecht-Nielsen R. , "Neurocomputing", pp. 122 124, Addison-Wesley Publishing Company.

[4] Jutten C. ,"Calcul neuromimetique et traitement du signal: Analyse en composants independents", $\mathrm{Ph}$. Tesis, in french, INPG-USMG Grenoble, 1987.

[5] Jutten C, Herault J. ,"Blind separation of sources, Part I: An adptive algorithm based on neuromimetic architecture", Signal Processing, vol 24, $n^{2} 1, \mathrm{pp}$. 1-10, July 1991

[6] Jutten C. ,Nguyen Thi, Dijkstra E. , Vittoz E. , Caelen J., "Blind Separation of Sources: an Algorithm for Separation of Convolutive Mixtures", Proc. Signal Processing Workshop on High Order Statistics, pp 275-278, Chanrrouse, Francia, July 1991

[7] Kolmogorov A. N. , "On the representation of continuous functions of many variables by superposition of continuous functions of one variable and addition". Dokl. Akad Nauk (USSR), in Russian, 114, 952-956, 1957.

[8] Kowalczyk A. , "Can multilayer mapping networks with finite number of real parameters harness the computatational power of Kolmogorov's representation theorem?",1991 IEEE International Joint Conference on Neural Networks, Singapore, pp. 2722-2728, 18-21 Nov. 1991

[9] Kurková V. , "Kolmogorov's Theorem is Relevant", Neural Computation (Winter 1991), vol. 3, no. 4, pp 617-622.

[10] Kurková V. , "Kolmogorov's Theorem and multilayer neural networks", Neural Networks, 1992, vol. 5, no. 3, pp 501-506.

[11] Lagunas M. A. ,Pagés A. , "Multitone tracking with coupled EKFs and high order learning". Proc. ICASSP-92, pp. V153-V156, San Francisco, USA.

[12] Lagunas M. A. , Pérez-Neira A. , "EKF schemes in array processing", NATO-ASI, Acoustic Signal Processing for Ocean Exploration, Madeira, July 26-August 7, 1992.

[13] Lagunas M. A., Pérez-Neira A. , Nájar M. , Pagés A. , "The Kolmogorov Signal Processor", IWANN' 93, Barcelona, Spain, 9-11 June, 1993.

[14] Lorentz G. , "Approximation of functions", pp. 168-179, Chelsea Publishing Co., New York, 1986.

[15] Nájar M. , Lagunas M. A. , Pérez-Neira A. , "Source Separation based on coupled single DOA Estimation Processors", Proc. ICASSP-93, Minneapolis, April 1993.

[16] Pérez-Neira A. ,Lagunas M. A. , "Array Covariance error measurement in adaptive source parameter estimation", Proc. Sixth SSAP Workshop on Statistical Signal \& Array Processing. October 7-9, 1992. 


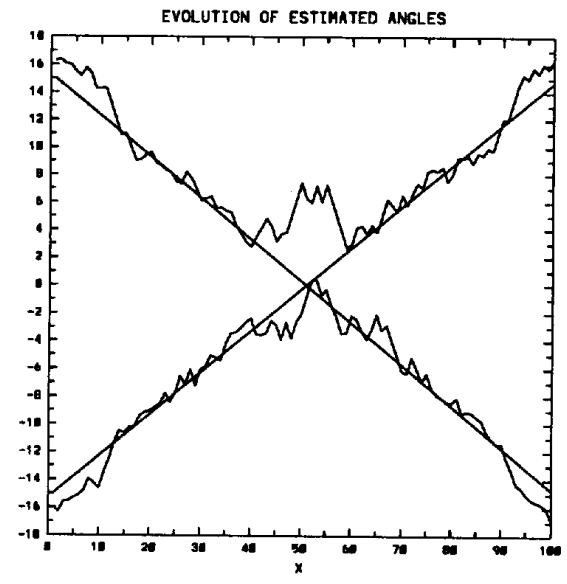

Figure 5.a.

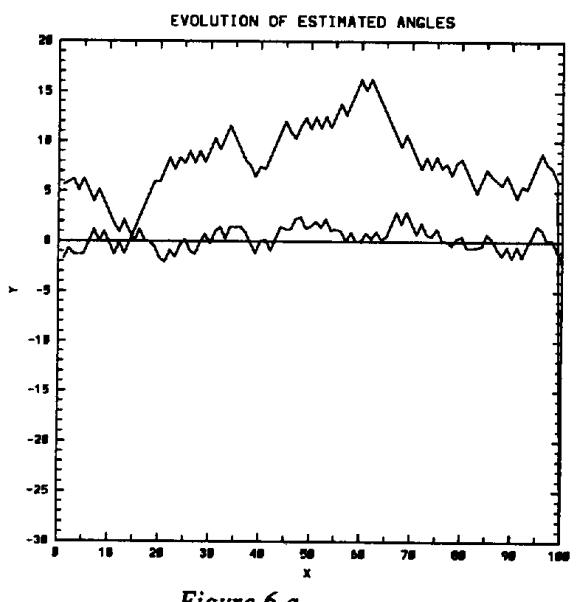

Figure 6.a.

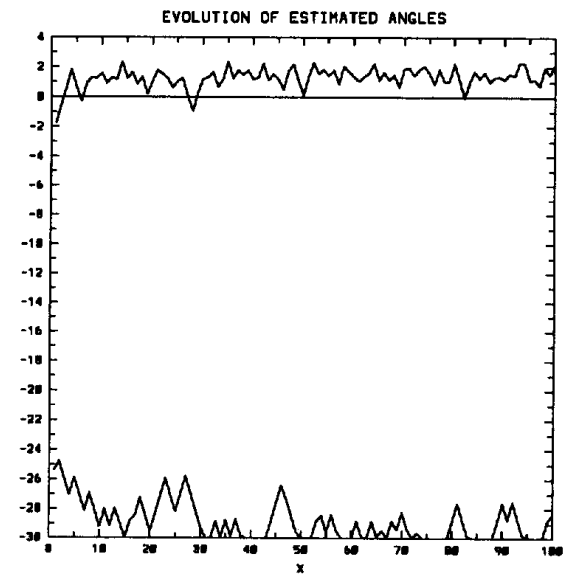

Figure 7.a.

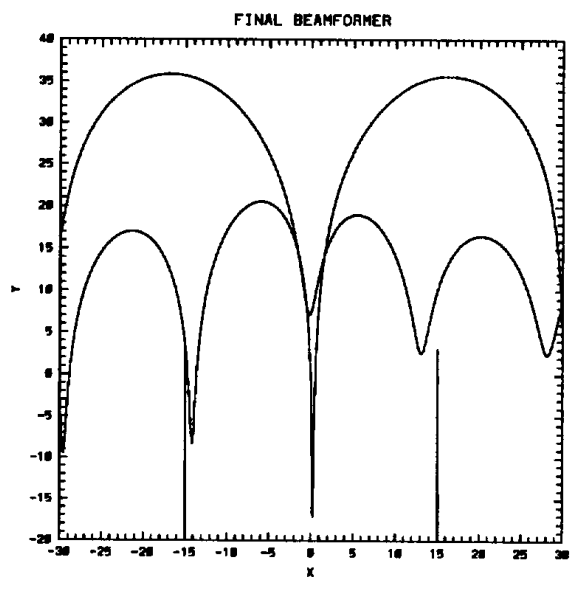

Figure 5.b.

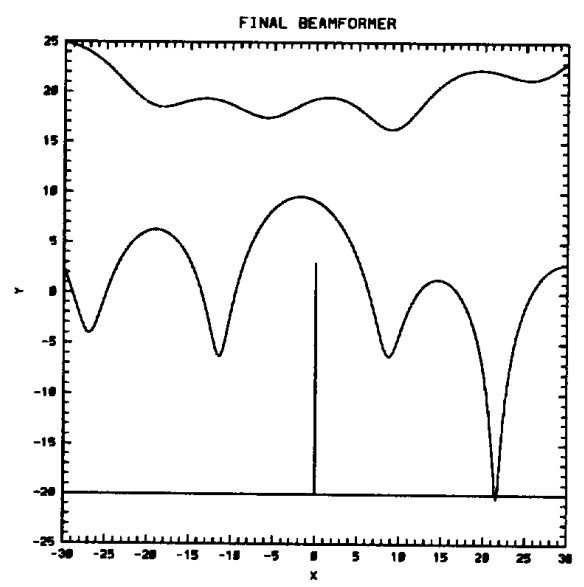

Figure 6.b.

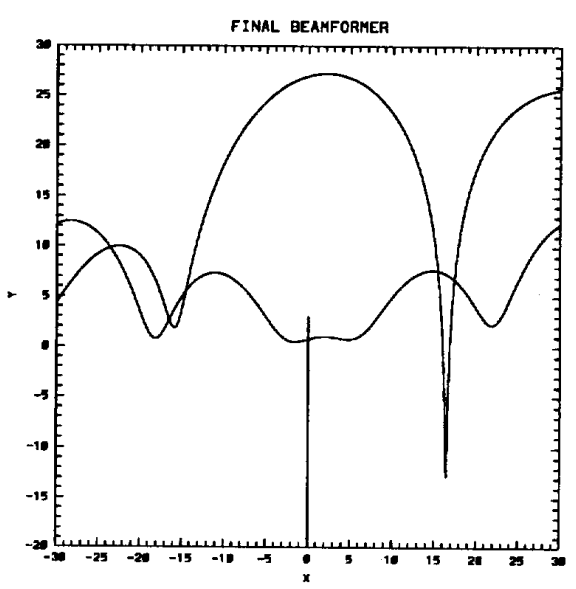

Figure 7.b. 